\title{
Mechanical Unfolding and Thermal Refolding of Single-Chain Nanoparticles Using Ligand-Metal Bonds
}

\author{
Avishai Levy, Roi Feinstein and Charles E. Diesendruck* \\ Schulich Faculty of Chemistry, Technion - Israel Institute of Technology, Haifa, 32000, Israel. \\ *To whom all correspondence should be addressed. Phone: (+972) 77-8873752. \\ Fax: (+972) 77-8873752. E-mail: charles@technion.ac.il
}

\section{Table of Contents}

$\begin{array}{ll}\text { I. General Experimental Details } & \text { S2 }\end{array}$

$\begin{array}{ll}\text { II. } & \text { Experimental Procedures }\end{array}$

III. Characterisation \& Results

$\begin{array}{ll}\text { IV. Statistical Analysis } & \text { S13 }\end{array}$

$\begin{array}{lll}\text { V. References } & \text { S14 }\end{array}$ 


\section{General Experimental Details}

All materials, unless otherwise stated, were purchased from commercial sources and used as received. Monomers and THF were purified by filtration through basic alumina column.

GPC analyses were done in THF at $30^{\circ} \mathrm{C}$ using a Thermo LC system equipped with Tosoh's TSKgel Guard Column HHR-L and four TSKgel G4000HHR columns in sequence. Detection was obtained with a penta-detector system which includes a Dionex DAD-3000 PDI UV-Vis Detector, Wyatt Viscostar II viscometer, Wyatt OPTILAB T-rEX refractometer, a Wyatt MALS DAWN HELEOS II 8+TR, and an online WyattQELS DLS. Wyatt's Astra 7.1.4 software was used for GPC data analysis and polymer properties calculation (molecular weights, PDIs, intrinsic viscosities and hydrodynamic radii).

Ultrasonication was done using in house-made Suslick cells connected to a SONICS Vibra-cell ultrasonic processor (500 Watt), tuned to $9.57 \mathrm{~W} \cdot \mathrm{cm}^{-2}$. All NMR spectra were recorded using Bruker AVANCE 200 or $300 \mathrm{MHz}$ or AVANCE III 400 or $600 \mathrm{MHz}$ spectrometers at the Technion NMR facilities.

The uncertainty reported in this study is not the full experimental uncertainty, but rather the statistical uncertainty based on noise levels of the various signals and repeated measurements. 


\section{Experimental Procedures}

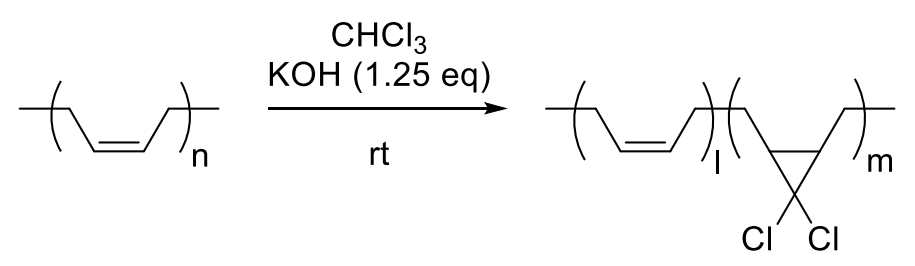

\section{Modified linear PB synthesis ${ }^{1}$}

Commercial cis-PB (2 g, $0.037 \mathrm{~mol}$ of double bonds) was fully dissolved in chloroform (30 mL, $0.37 \mathrm{~mol})$, together with benzyltriethylammonium chloride $(0.7 \mathrm{mg}, 0.66 \mathrm{mmol}) . \mathrm{NaOH}$ (1.85 g, $0.046 \mathrm{~mol})$ was then added in small portions. The reaction mixture was stirred at room temperature overnight. The mixture was washed with water, brine and water again, before addition of magnesium sulphate as a drying agent. The mixture was concentrated using a rotovap and the polymer isolated by precipitation in methanol and drying under high vacuum. Modified copolymer $\mathbf{L}$ was obtained (2.7 g, $78.5 \%$ yield) with $45 \%$ of the monomers converted to gemdichlorocyclopropane. Composition of the copolymer was determined by ${ }^{1} \mathrm{H}-\mathrm{NMR}$ spectroscopy (see part III).

\section{Procedure for intramolecular collapse ${ }^{2}$}

CL4 (5\% molar cross-linked polymer) is used as an example. Modified PB copolymer $\mathbf{L}$ (10 mg, $1.094 \cdot 10^{-4} \mathrm{~mol}$ total monomers, $5.5 \mu \mathrm{mol} 5 \%$ of monomers, $1 \mathrm{eq}$ ) was dissolved in dry THF $(5 \mathrm{~mL})$ the night before the desired experiment. Then, BHT $(0.2 \mathrm{mg}$, using $0.2 \mathrm{~mL}$ of 1 $\mathrm{mg} / \mathrm{mL}$ stock solution in dry THF), and the additional amount of dry THF were added (4.28 $\mathrm{mL}$ in this example) to react a final solution volume of $10 \mathrm{~mL}$ (polymer concentration $1 \mathrm{mg} / \mathrm{ml}$ ). chlorobis(ethylene)rhodium dimer $(0.52 \mathrm{mg}, 1.37 \mu \mathrm{mol}, 0.25 \mathrm{eq})$ was added dropwise to the stirred solution via syringe using a fresh stock solution $(1 \mathrm{mg} / \mathrm{mL})$ which was prepared as follows: dry THF (3 mL) was added to chlorobis(ethylene)rhodium dimer ( $3 \mathrm{mg}, 7.88 \mu \mathrm{mol})$ which was immediately vortexed for $5 \mathrm{sec}$ and filtered using a syringe filter $(0.022 \mu \mathrm{m})$. The mixture was stirred for $3 \mathrm{~h}$ and used immediately in sonication experiments. 


\section{Sonication experiments of polymer $L$}

Polymer $\mathbf{L}$ was dissolved in THF (1 $\mathrm{mg} / \mathrm{mL})$ and BHT (2\%) was added. The solution was then transferred to an oven-dried Suslick cell, which was placed into the sonicator collar and screwed onto the probe. The Suslick cell was lowered into acetone $\left(-9{ }^{\circ} \mathrm{C}\right)$ bath, and $\mathrm{N}_{2}$ was sparged for 15 min prior to ultrasound irradiation and continued throughout the experiment. Pulsed sonication ( $1 \mathrm{~s}$ on, $2 \mathrm{~s}$ off) was carried out using $20 \%$ amplitude $\left(9.57 \mathrm{~W} \cdot \mathrm{cm}^{-2}\right)$ for $15 \mathrm{~min}$ (net sonication time). The sonicated solution was evaporated and the polymer dissolved in $\mathrm{CDCl}_{3}$. Gem-DCC activation was quantified by ${ }^{1} \mathrm{H}-\mathrm{NMR}$ spectroscopy.

\section{Sonication experiments of polymers CL1-7}

The procedure is similar to sonication of $\mathbf{L}$, but with the following changes. The polymer solution was sonicated immediately at the end of the $3 \mathrm{~h}$ intramolecular collapse reaction. After sonication, 2-diphenylphosphinobenzaldehyde (4 eq.) was added to extract the rhodium and allow solvent evaporation without cross-linking. In the refolding experiments, the pulsed program was changed, according to the experiment ( $1 \mathrm{~s}$ on, $1 \backslash 2 \backslash 4 \backslash 8 \backslash 16 \mathrm{~s}$ off) to a total of $15 \mathrm{~min}$ net sonication time. Gem-DCC activation was quantified by ${ }^{1} \mathrm{H}-\mathrm{NMR}$ spectroscopy. 


\section{Characterization \& Results}

Table S1. Characterization of polymers used in this study.

\begin{tabular}{|l|c|c|c|c|c|c|}
\hline Polymer & $\begin{array}{c}\text { CL ratio (\% } \\
\text { mol })^{\mathrm{a}}\end{array}$ & $\begin{array}{c}\text { Retention } \\
\text { volume }(\mathrm{mL})^{\mathrm{b}}\end{array}$ & $\begin{array}{c}\text { Mn (calculated) } \\
(\mathrm{kDa})\end{array}$ & $\mathrm{Mn}^{(\mathrm{kDa})^{\mathrm{c}}}$ & PDI $^{\mathrm{c}}$ & $\begin{array}{c}\mathrm{R}_{\mathrm{h}} \\
(\mathrm{nm})^{\mathrm{c}}\end{array}$ \\
\hline L & 0 & 23.9 & - & 172.7 & 1.4 & 16.6 \\
\hline CL1 & 0.5 & 25.5 & 173.9 & 173.5 & 1.3 & 16.0 \\
\hline CL2 & 1 & 26.5 & 174.9 & 173.9 & 1.3 & 14.6 \\
\hline CL3 & 3 & 29.3 & 179.3 & 177.9 & 1.2 & 12.1 \\
\hline CL4 & 5 & 30.4 & 183.7 & 180.3 & 1.1 & 11.8 \\
\hline CL5 & 8 & 31.3 & 190.3 & 188.4 & 1.1 & 7.1 \\
\hline CL6 & 10 & 32.7 & 194.7 & 191.8 & 1.1 & 6.3 \\
\hline CL7 & 15 & 32.2 & 205.7 & 198.7 & 1.1 & 5.4 \\
\hline
\end{tabular}

[a] Calculated from relative amount of Rh complex added. [b] Peak maximum $-1 \mathrm{~mL} / \mathrm{min}$ THF. [c] Calculated from triple-detector GPC. PDI is underestimated as typical for triple-detector GPC.

\section{NMR spectra of polymers}

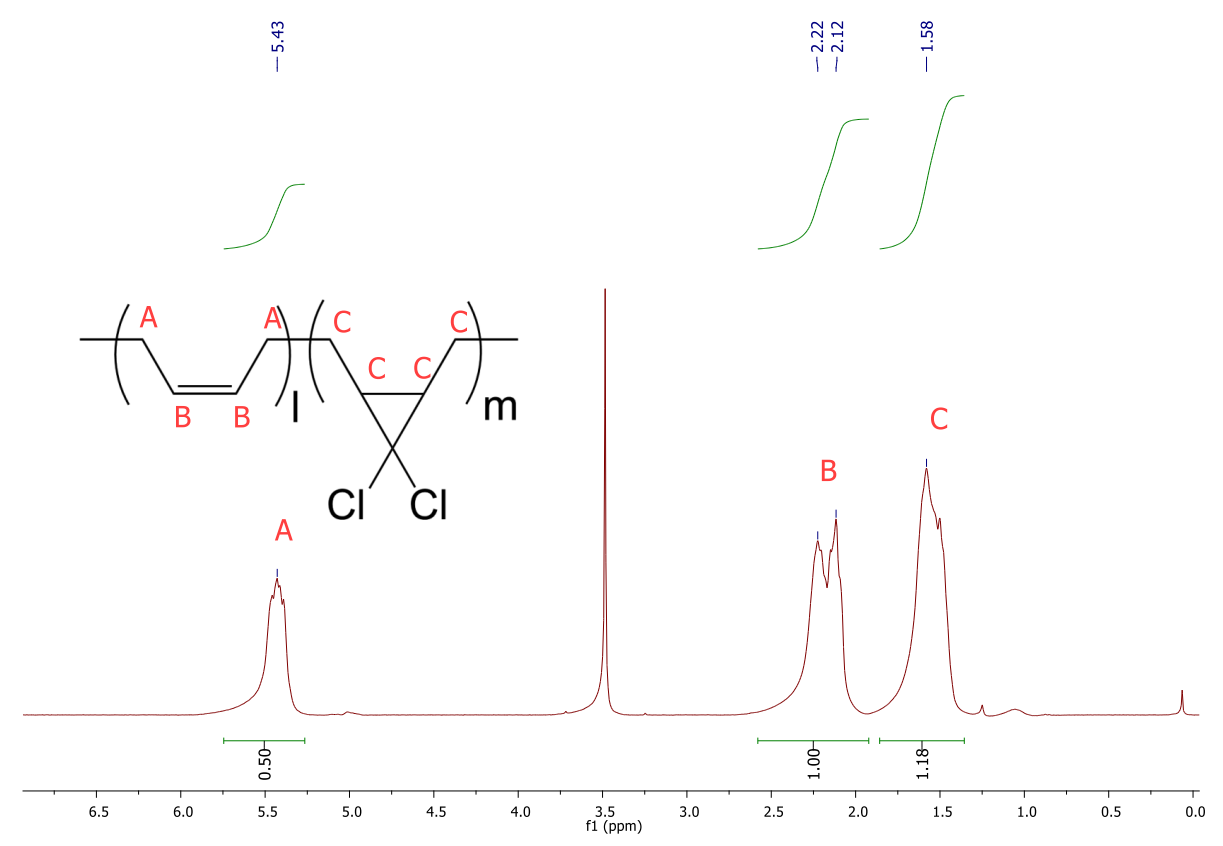

Figure S1. ${ }^{1} \mathrm{H}-\mathrm{NMR}$ spectrum of $\mathbf{L}$. 


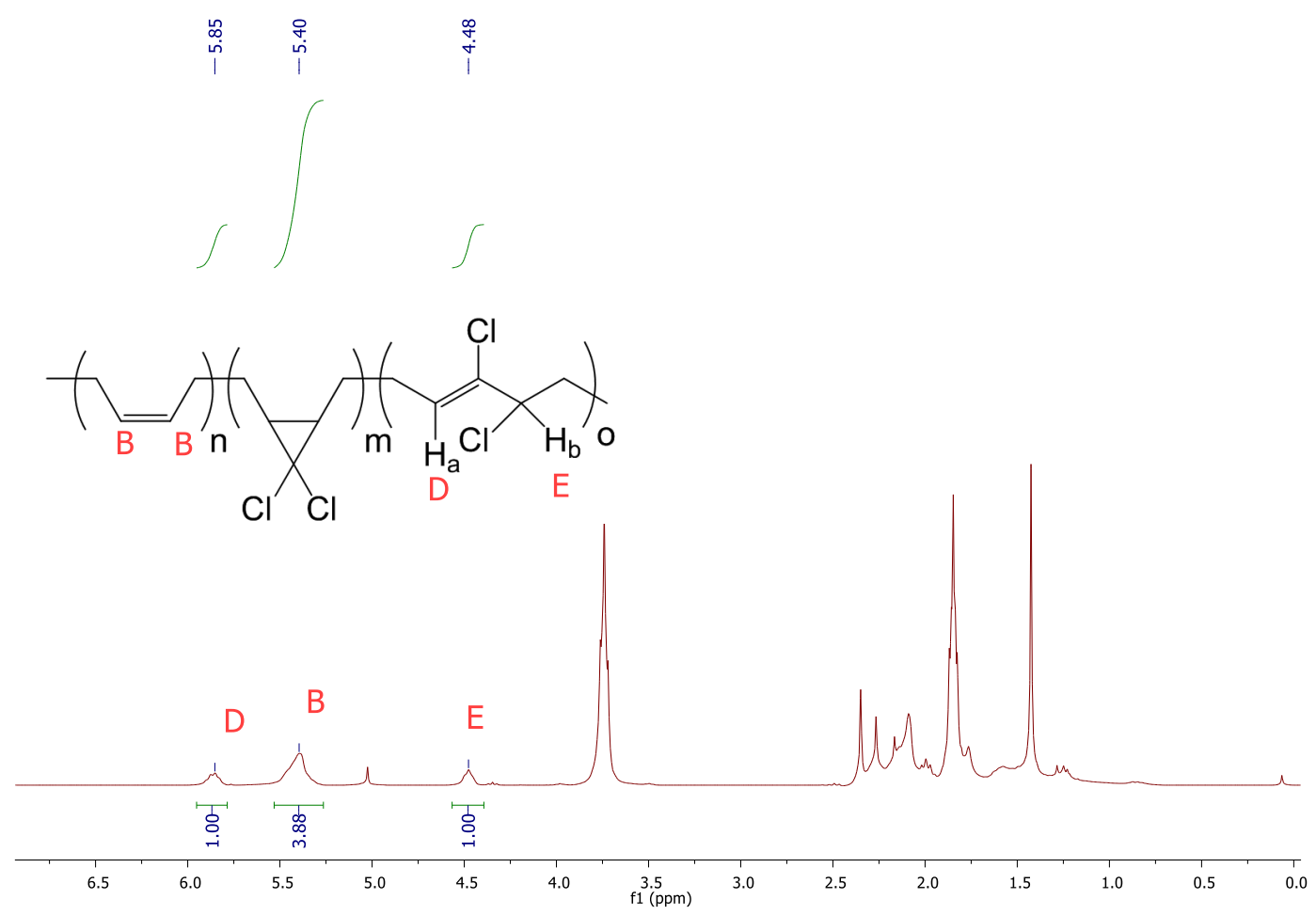

Figure S2. ${ }^{1} \mathrm{H}-\mathrm{NMR}$ spectrum of $\mathbf{L}$ copolymer after sonication.

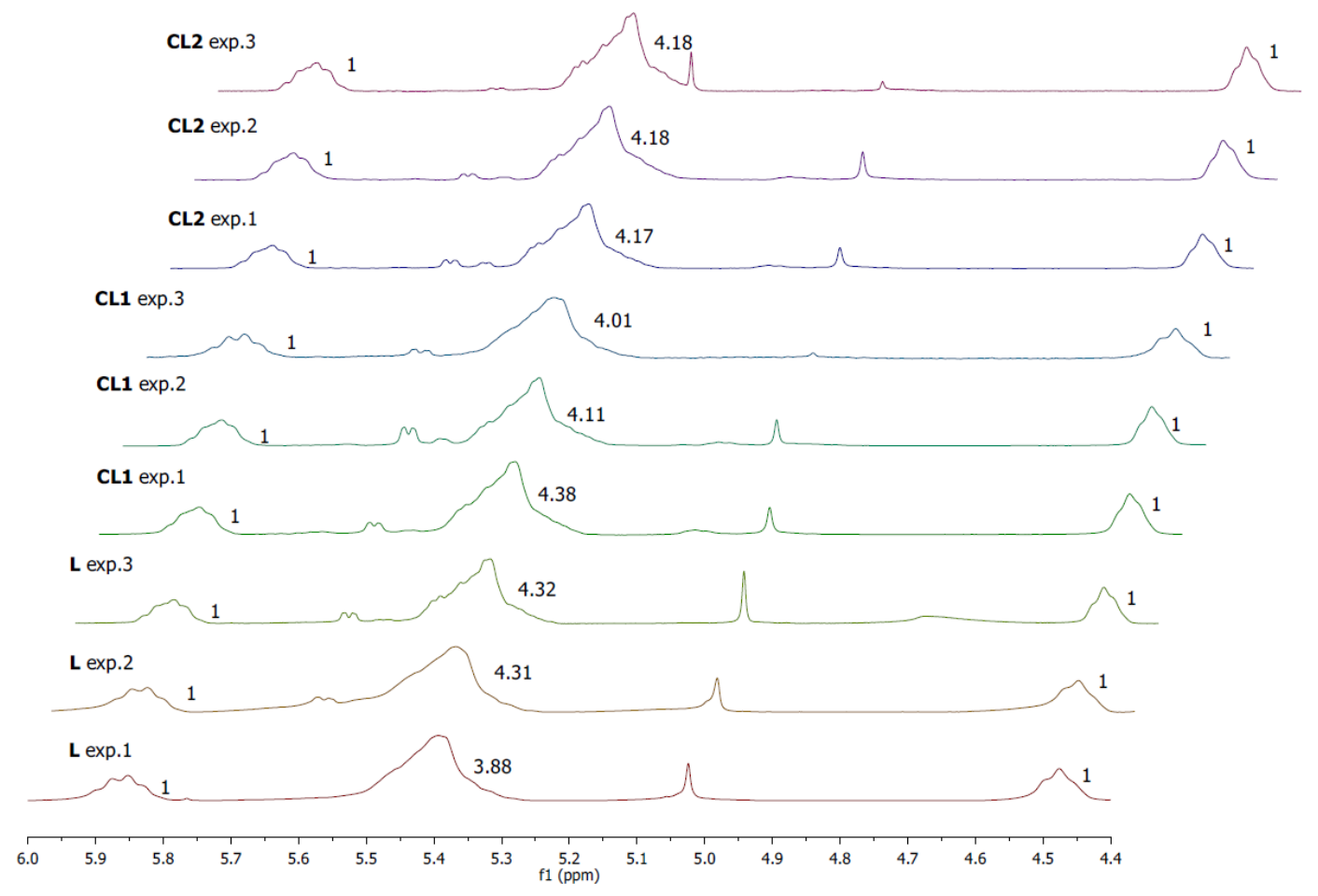

Figure S3. Double-bond region in ${ }^{1} \mathrm{H}-\mathrm{NMR}$ spectra of $\mathbf{L}, \mathbf{C L 1}$ and $\mathbf{C L 2}$ after sonication. 


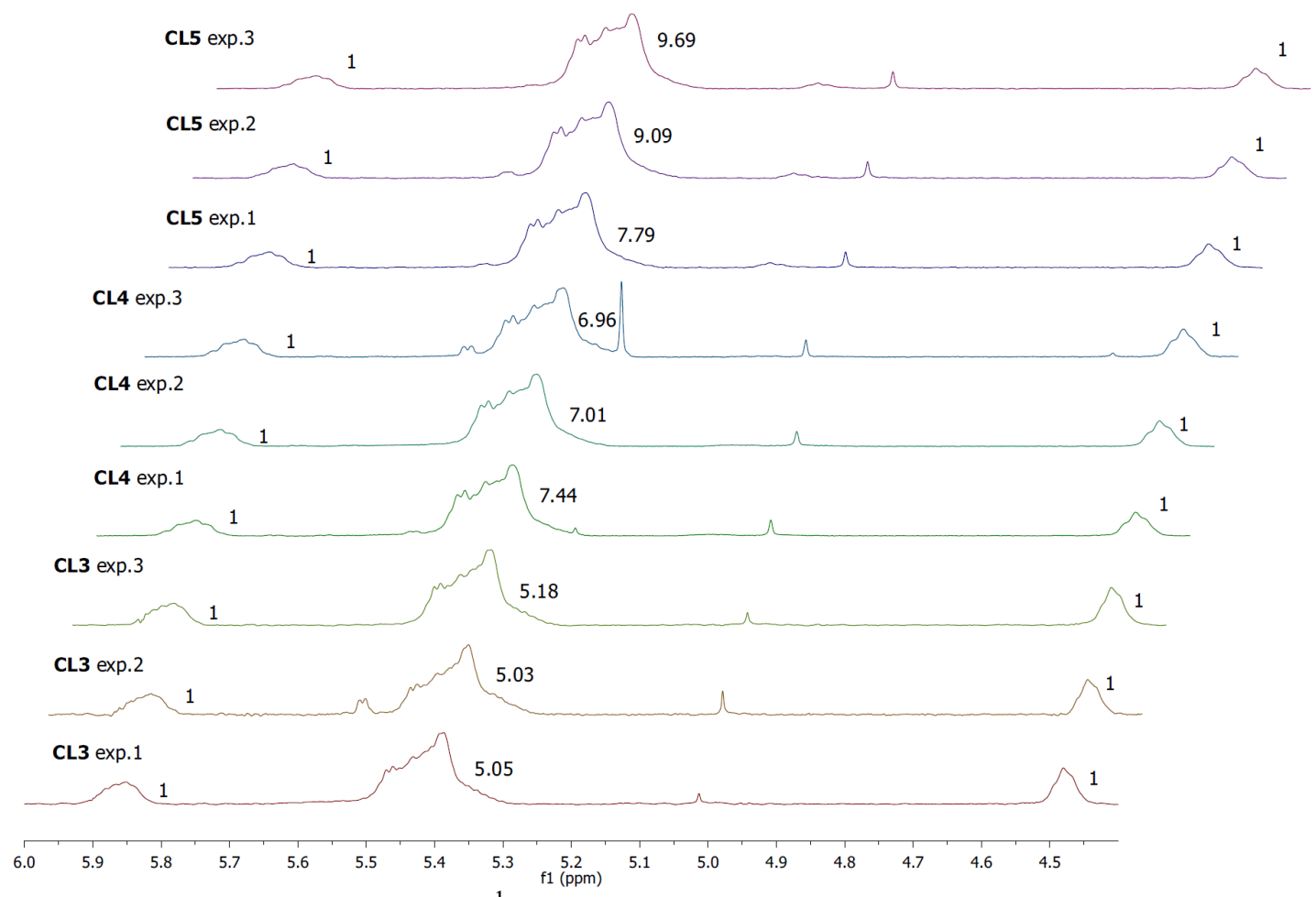

Figure S4. Double-bond region in ${ }^{1} \mathrm{H}-\mathrm{NMR}$ spectra of CL3, CL4 and CL5 after sonication.

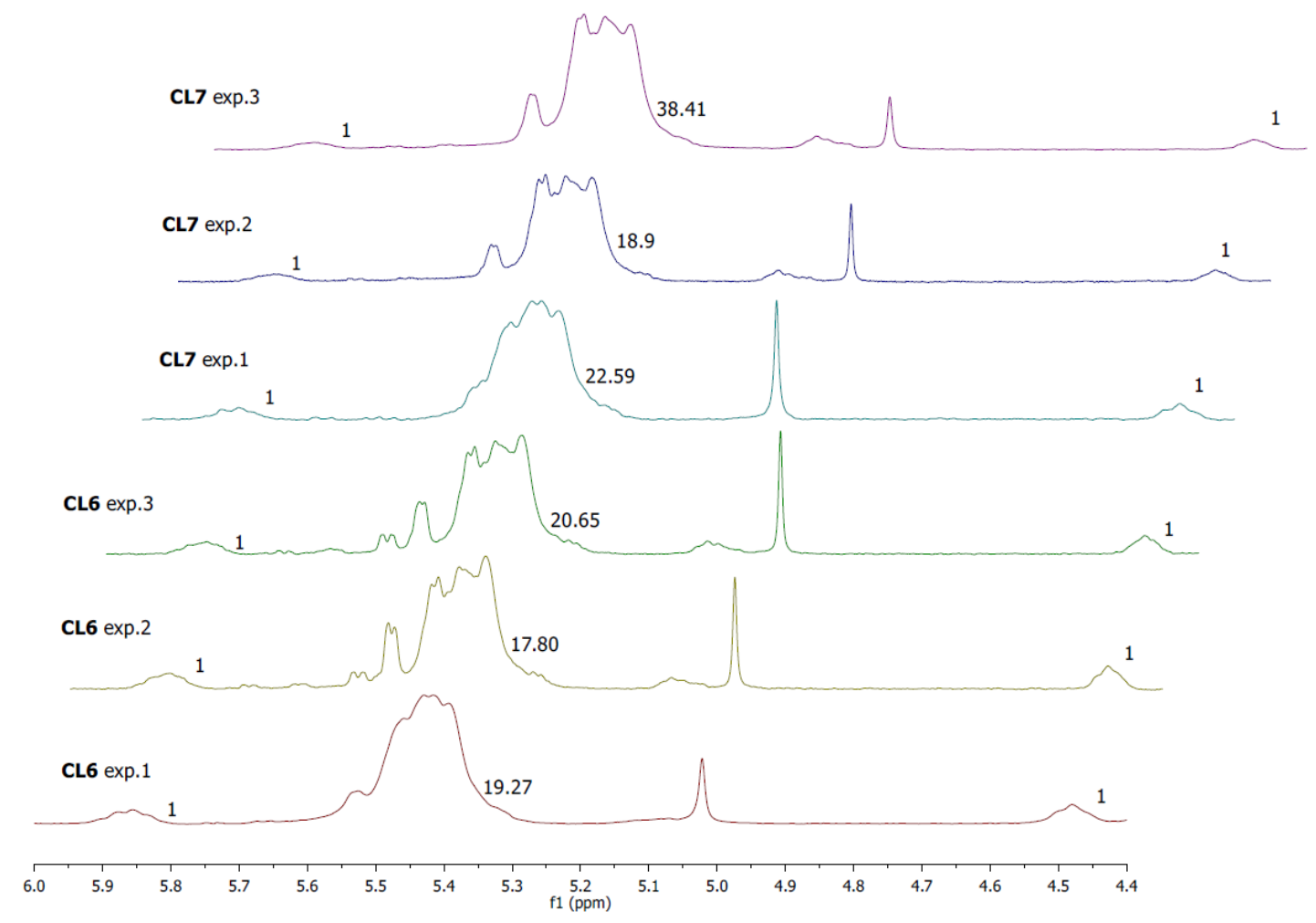

Figure S5. Double-bond region in ${ }^{1} \mathrm{H}-\mathrm{NMR}$ spectra of CL6 and CL7 after sonication. 


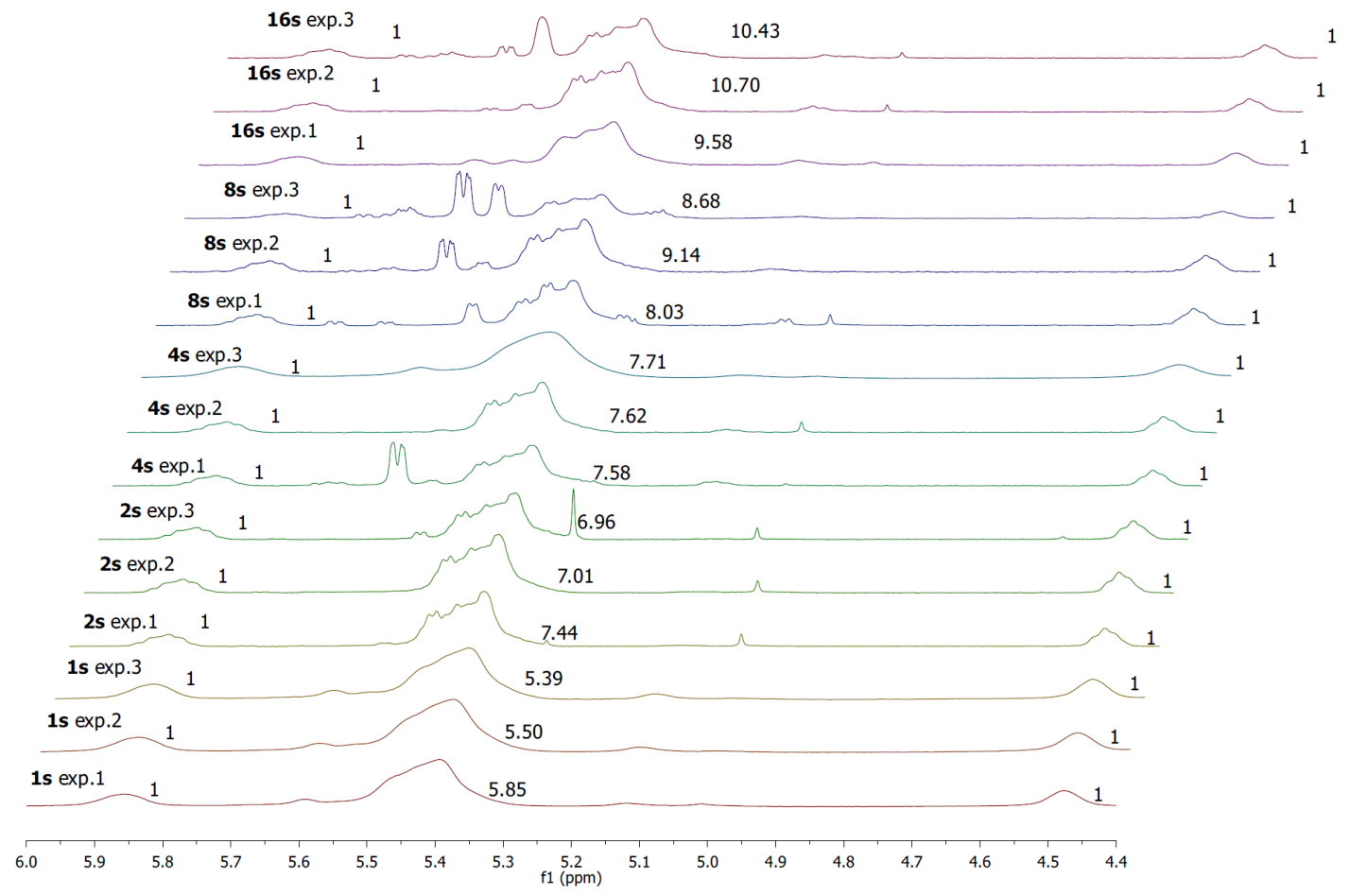

Figure S6. Double-bond region in ${ }^{1} \mathrm{H}-\mathrm{NMR}$ spectra of CL4 after sonications with different delays.

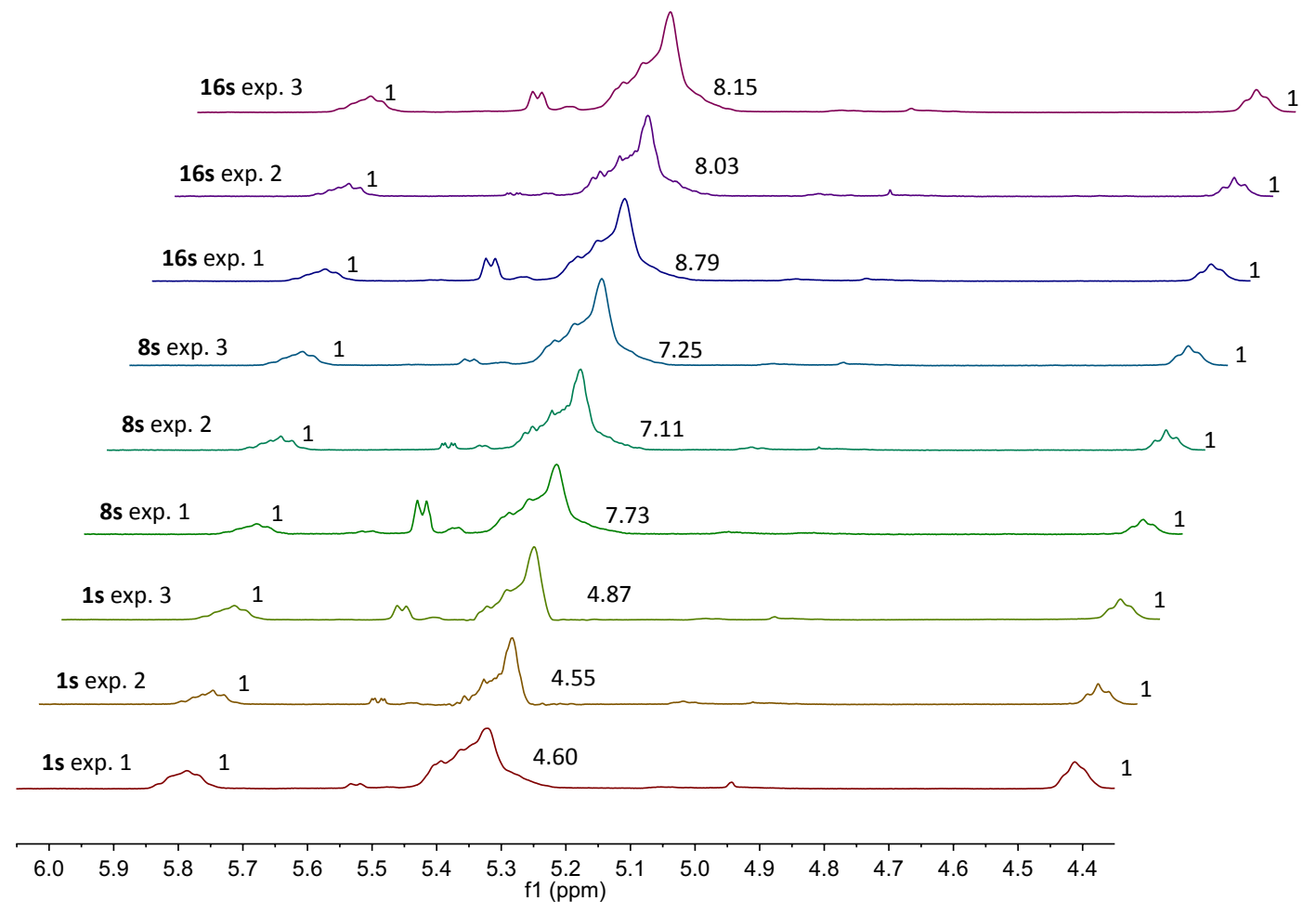

Figure S7. Double-bond region in ${ }^{1} \mathrm{H}-\mathrm{NMR}$ spectra of $\mathbf{C L 3}$ after sonications with different delays. 


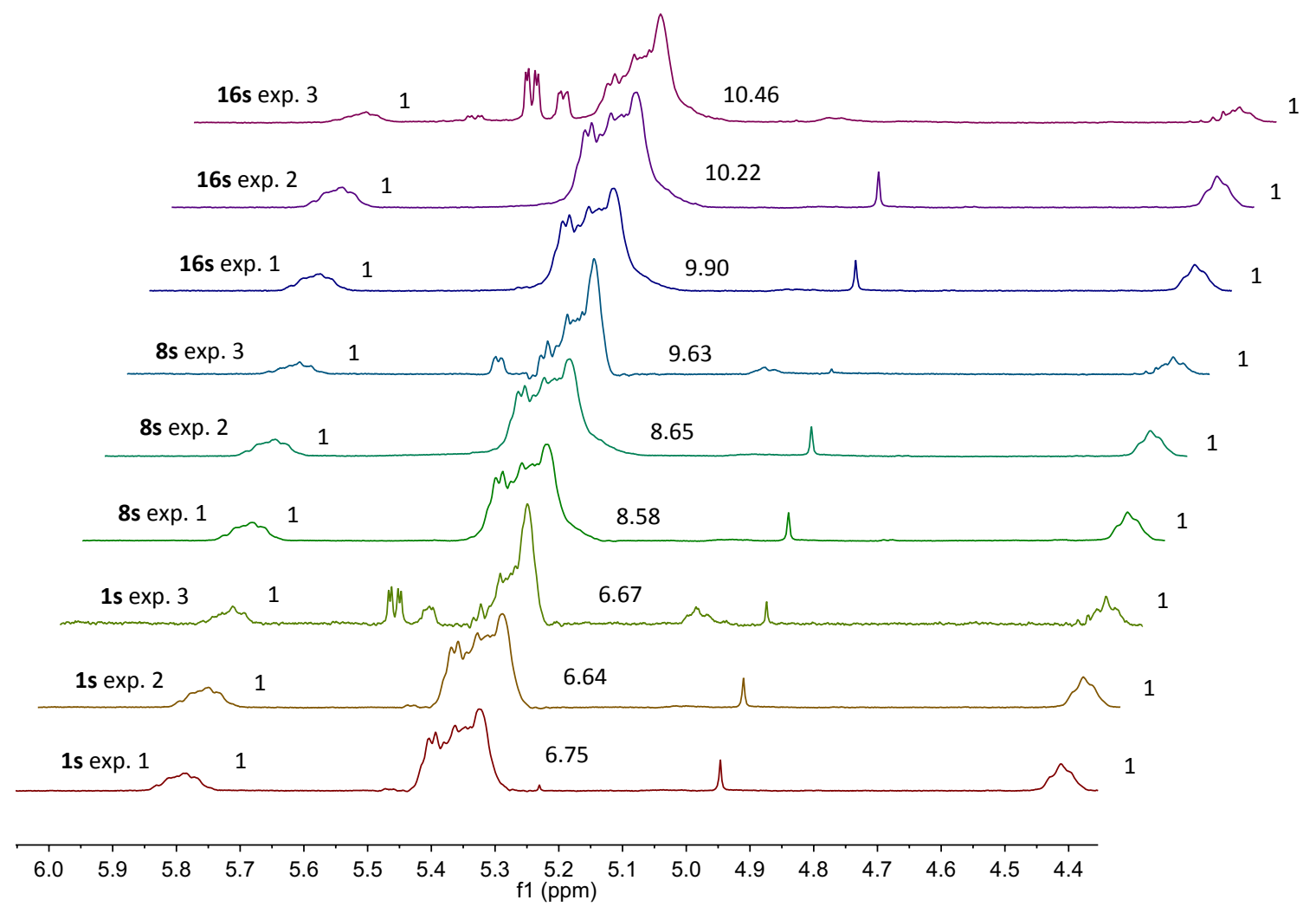

Figure S8. Double-bond region in ${ }^{1} \mathrm{H}-\mathrm{NMR}$ spectra of CL5 after sonications with different delays.

\section{Monomer composition in $\mathrm{L}$}

The monomer composition in $\mathbf{L}$ was calculated from ${ }^{1} \mathrm{H}-\mathrm{NMR}$ spectrum according to the peak assignment reported in the literature, ${ }^{3}$ using integration and the following equation:

$$
\begin{gathered}
\% n(\text { gem }-D C C)=\frac{I_{\text {perH } \text { gem }-D C C}}{I_{\text {per } H \text { total }}} \times 100 \\
I_{\text {perH } \text { gem }-D C C}=\frac{I_{C}}{6 H}=\frac{1.18}{6}=0.197 \\
I_{\text {per } H 1,4-\text { butadiene }}=\frac{I_{A}+I_{B}}{6 H}=\frac{0.5+1}{6}=0.25 \\
I_{\text {per H total }}=I_{\text {perH } \text { gem-DCC }}+I_{\text {perH } \text { gem }-D C C}=0.197+0.25=0.446 \\
\% n(\text { gem }-D C C)=\frac{I_{\text {perH gem-DCC }}}{I_{\text {per } H \text { total }}} \times 100=\frac{0.197}{0.446} \times 100=45 \%
\end{gathered}
$$




\section{Calculation of required metal quantities for the cross-linking reaction}

Calculations are based on the comonomer composition obtained from NMR spectra, as described above. $10 \mathrm{mg}$ of polymer $\mathbf{L}$ was used for each cross-linking reaction. As an example, the calculation of the cross-linking reaction of CL7 (15\% cross-linked) is presented:

$$
\begin{gathered}
n_{\text {monomers }}=n_{\text {total }} \cdot \% n(1,4 \text { butadiene }) \cdot M w_{1,4 \text { butadiene }}+n_{\text {total }} \cdot \% n(\mathrm{gem}- \\
D C C) \cdot M w_{\text {gem-DCC }}=0.01 \mathrm{gr} \\
n_{\text {monomers }}=n_{\text {total }} \cdot 0.55 \cdot 55.54 \frac{\mathrm{gr}}{\mathrm{mol}}+n_{\text {total }} \cdot 0.45 \cdot 137.01 \frac{\mathrm{gr}}{\mathrm{mol}}=0.01 \mathrm{gr} \\
n_{\text {monomers }}=1.10 \cdot 10^{-4} \mathrm{~mol} \\
n_{15 \% \text { monomers }}=1.10 \cdot 10^{-4} \mathrm{~mol} \cdot 15 \%=1.64 \cdot 10^{-5} \mathrm{~mol} \\
n_{\text {Rh Complex }}=\frac{n_{15 \% \mathrm{monomers}}}{4}=4.11 \cdot 10^{-6} \mathrm{~mol} \\
m_{\text {Rh Complex }}=4.11 \cdot 10^{-6} \mathrm{~mol} \cdot 380.87 \frac{\mathrm{gr}}{\mathrm{mol}}=1.56 \mathrm{mg}
\end{gathered}
$$

\section{Gem-DCC activation quantification}

Determination of gem-DCC activation was done based on ${ }^{1} \mathrm{H}-\mathrm{NMR}$ spectra. ${ }^{3}$ The calculation assumes that, if $100 \%$ of the gem-DCC were activated, the ratio between activated chloroalkene and non-substituted double bonds would be equal to the ratio of gem-DCC units and nonsubstituted double bonds in polymer $\mathbf{L}$ before sonication.

$$
\% \text { gem }- \text { DCC activtion }=\frac{r_{\text {activated gem-DCC to 1,4 butadiene }}}{r_{\text {gem-DCC to 1,4 butadiene }}} \times 100
$$

From the comonomer composition, the ratio between gem-DCC units and double bonds in polymer $\mathbf{L}$ before sonication is:

$$
r_{\text {gem-DCC to 1,4 butadiene }}=\frac{I_{\text {perH } \text { gem-DCC }}}{I_{\text {per } H \text { 1,4-butadiene }}}=\frac{0.197}{0.25}=0.8
$$


The calculations of gem-DCC activation in polymer $\mathbf{L}$ after the $3^{\text {rd }}$ sonication experiment is shown as an example:

$$
\begin{gathered}
I_{\text {perH activated gem-DCC }}=\frac{I_{D \text { or } E}}{1 H}=\frac{1}{1}=1 \\
I_{\text {per H 1,4-butadiene }}=\frac{I_{B}}{2 H}=\frac{4.33}{2}=2.16 \\
r_{\text {activated gem-DCC to 1,4 butadiene }}=\frac{I_{\text {perH activated gem }-D C C}}{I_{\text {per } H \text { 1,4-butadiene }}}=\frac{1}{2.165}=0.46 \\
\% G e m-D C C \text { activtion }=\frac{0.46}{0.8} \times 100=58 \%
\end{gathered}
$$

\section{Intramolecular collapse reaction time}

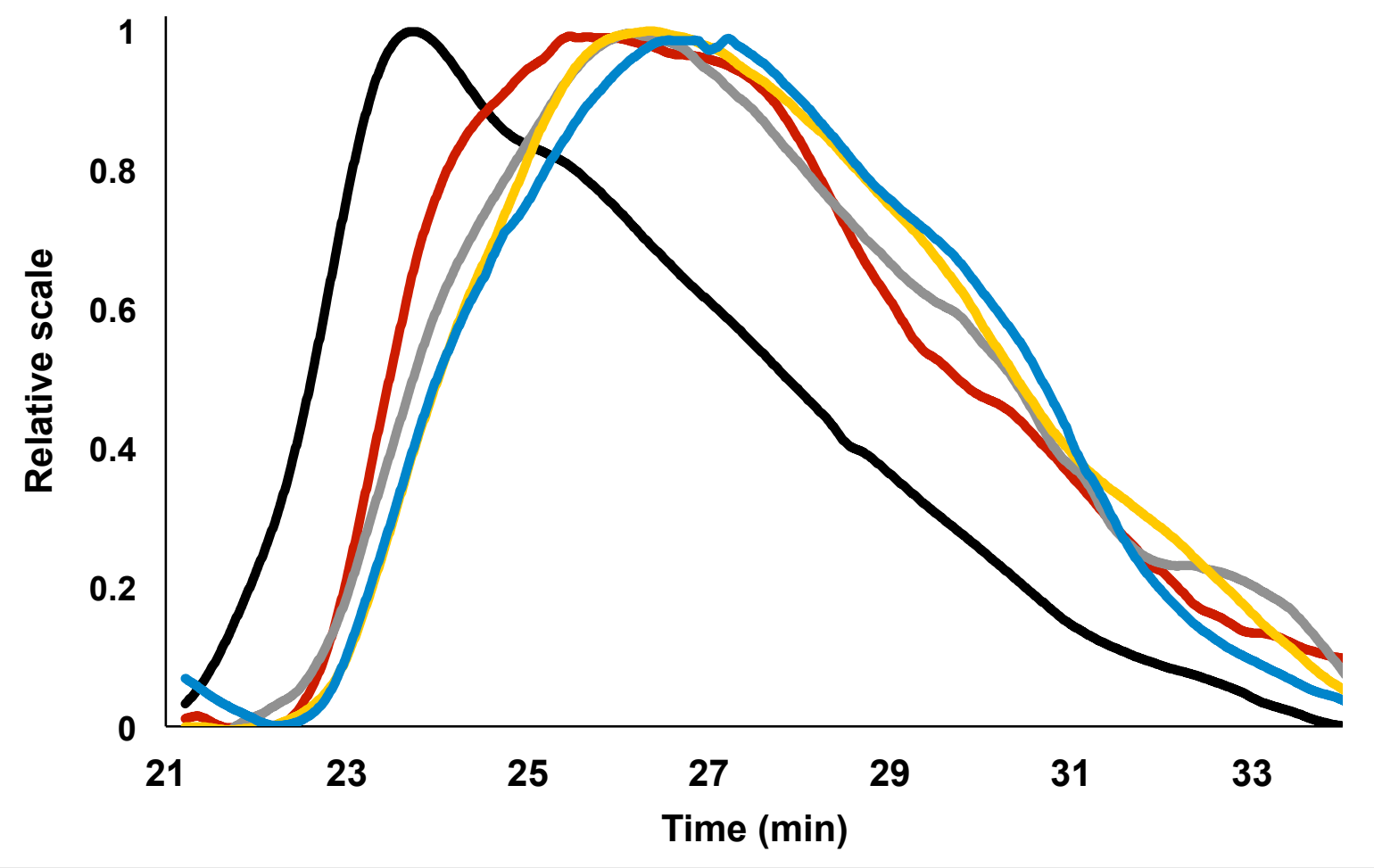

Figure S9. GPCs of CL1 during intramolecular collapse after 0 min (black), 5 mins (red), $1 \mathrm{hr}$ (grey), $3 \mathrm{hrs}$ (yellow) and $24 \mathrm{hrs}$ (light blue) in THF. Signal shown from differential refractiveindex detector. 


\section{Sonication results}

The gem-DCC activation rates were calculated according to peak integration (Figures S3-S8) following the examples shown above.

The results of three sonication experiments for each polymer are summarized in Table S2.

Results of sonication experiments of polymer CL3-5 with different delays between pulses are summarized in Table S3-5.

Table S2. Gem-DCC activation rates after sonication.

\begin{tabular}{|c|c|c|c|c|c|c|c|c|}
\hline Polymer & L & CL1 & CL2 & CL3 & CL4 & CL5 & CL6 & CL7 \\
\hline Experiment 1 & 64.43 & 59.67 & 57.08 & 49.50 & 33.60 & 32.09 & 12.97 & 11.07 \\
\hline Experiment 2 & 58.00 & 59.81 & 60.83 & 49.70 & 35.66 & 27.50 & 14.04 & 13.23 \\
\hline Experiment 3 & 57.87 & 59.95 & 62.34 & 48.26 & 35.92 & 25.80 & 12.11 & 6.51 \\
\hline Average & 60.10 & 59.81 & 60.08 & 49.16 & 35.06 & 28.46 & 13.04 & 10.27 \\
\hline Standard deviation & 3.75 & 0.14 & 2.71 & 0.78 & 1.27 & 3.25 & 0.97 & 3.43 \\
\hline
\end{tabular}

Table S3. Gem-DCC activation rates after sonication of polymer CL4 with different delays between pulses.

\begin{tabular}{|c|c|c|c|c|c|}
\hline Interval [s] & $\mathbf{1 s}$ & $\mathbf{2 s}$ & $\mathbf{4 s}$ & $\mathbf{8 s}$ & $\mathbf{1 6 s}$ \\
\hline Experiment 1 & 45.45 & 33.60 & 32.98 & 31.13 & 26.10 \\
\hline Experiment 2 & 46.38 & 35.66 & 32.81 & 27.35 & 23.36 \\
\hline Experiment 3 & 42.74 & 35.92 & 32.43 & 28.80 & 23.97 \\
\hline Average & 44.86 & 35.06 & 32.74 & 29.10 & 24.48 \\
\hline Standard deviation & 1.90 & 1.27 & 0.28 & 1.91 & 1.43 \\
\hline
\end{tabular}

Table S4. Gem-DCC activation rates after sonication of polymer $\mathbf{C L 3}$ with different delays between pulses.

\begin{tabular}{|c|c|c|c|c|}
\hline Interval [s] & $\mathbf{1 s}$ & $\mathbf{2 s}$ & $\mathbf{8 s}$ & $\mathbf{1 6 s}$ \\
\hline Experiment 1 & 54.34 & 49.50 & 32.34 & 28.44 \\
\hline Experiment 2 & 54.94 & 49.70 & 35.16 & 31.13 \\
\hline Experiment 3 & 51.33 & 48.26 & 34.48 & 30.67 \\
\hline Average & 53.54 & 49.16 & 34.00 & 30.08 \\
\hline Standard deviation & 1.94 & 0.78 & 1.47 & 1.44 \\
\hline
\end{tabular}


Table S5. Gem-DCC activation rates after sonication of polymer CL5 with different delays between pulses.

\begin{tabular}{|c|c|c|c|c|}
\hline Interval [s] & $\mathbf{1 s}$ & $\mathbf{2 s}$ & $\mathbf{8 s}$ & $\mathbf{1 6 s}$ \\
\hline Experiment 1 & 37.04 & 32.09 & 29.14 & 25.25 \\
\hline Experiment 2 & 37.65 & 27.50 & 28.25 & 24.46 \\
\hline Experiment 3 & 36.93 & 25.80 & 25.96 & 23.90 \\
\hline Average & 37.21 & 28.46 & 27.78 & 24.54 \\
\hline Standard deviation & 0.39 & 3.25 & 1.64 & 0.68 \\
\hline
\end{tabular}

\section{Statistical Analysis}

Statistical analysis was performed using a Student's t-test with a minimum confidence level of 0.05 for statistical significance and assuming equal sample sizes and unequal variance. All values are reported as the mean and standard deviation of the mean.

Table S6. Student $t$ test results comparing individual rate constants of every polymer pair in gem-DCC activation experiments. Red color indicates no significant difference between the pair.

\begin{tabular}{|c|c|c|c|c|c|c|c|}
\hline & CL1 & CL2 & CL3 & CL4 & CL5 & CL6 & CL7 \\
\hline $\mathbf{L}$ & 0.9 & 0.9953 & 0.0078 & 0.0004 & 0.0004 & $<0.0001$ & $<0.0001$ \\
\hline CL1 & & 0.8699 & $<0.0001$ & $<0.0001$ & $<0.0001$ & $<0.0001$ & $<0.0001$ \\
\hline CL2 & & & 0.0026 & 0.0001 & 0.0002 & $<0.0001$ & $<0.0001$ \\
\hline CL3 & & & & $<0.0001$ & 0.0004 & $<0.0001$ & $<0.0001$ \\
\hline CL4 & & & & & 0.0308 & 0.0014 & 0.0003 \\
\hline CL5 & & & & & & 0.0014 & 0.0026 \\
\hline CL6 & & & & & & & 0.2495 \\
\hline
\end{tabular}

Table S7. Student $t$ test results comparing individual rate constants of every experiment pair in time-interval sonication experiments using $\mathbf{C L 4}$. Red color indicates no significant difference between the pair.

\begin{tabular}{|c|c|c|c|c|}
\hline & $2 s$ & $4 s$ & $8 s$ & $16 s$ \\
\hline $1 \mathrm{~s}$ & 0.0017 & 0.0004 & 0.0005 & 0.0001 \\
\hline $2 s$ & & 0.0367 & 0.0107 & 0.0007 \\
\hline $4 s$ & & & 0.0306 & 0.0006 \\
\hline $8 s$ & & & & 0.0286 \\
\hline
\end{tabular}


Table S8. Student t test results comparing individual rate constants of every experiment pair in time-interval sonication experiments using CL3. Red color indicates no significant difference between the pair.

\begin{tabular}{|c|c|c|c|}
\hline & $\mathbf{2 s}$ & $\mathbf{8 s}$ & $\mathbf{1 6 s}$ \\
\hline $\mathbf{1 s}$ & 0.0442 & 0.0002 & 0.0001 \\
\hline $\mathbf{2 n}$ & & 0.0005 & 0.0018 \\
\cline { 1 - 3 } & $\mathbf{8 s}$ & & 0.0302 \\
\hline
\end{tabular}

Table S9. Student $t$ test results comparing individual rate constants of every experiment pair in time-interval sonication experiments using CL5. Red color indicates no significant difference between the pair.

\begin{tabular}{|c|c|c|c|}
\hline & $\mathbf{2 s}$ & $\mathbf{8 s}$ & $\mathbf{1 6 s}$ \\
\hline $\mathbf{1 s}$ & 0.0415 & 0.0073 & $<0.0001$ \\
\cline { 1 - 2 } $\mathbf{2 s}$ & & 0.7617 & 0.1671 \\
\cline { 1 - 3 } $\mathbf{8 s}$ & & & 0.0594 \\
\hline
\end{tabular}

\section{References}

(1) Roberts, Gilbert, R. and W. No Title. In An Introduction to Modern Experiments Organic Chemistry; 1974.

(2) Mavila, S.; Diesendruck, C. E.; Linde, S.; Amir, L.; Shikler, R.; Lemcoff, N. G. Polycyclooctadiene Complexes of Rhodium(I): Direct Access to Organometallic Nanoparticles. Angew. Chem. Int. Ed. 2013, 52 (22), 5767-5770.

(3) Lenhardt, J. M.; Black, A. L.; Craig, S. L. Gem-Dichlorocyclopropanes as Abundant and Efficient Mechanophores in Polybutadiene Copolymers under Mechanical Stress. J. Am. Chem. Soc. 2009, 131 (31), 10818-10819. 